\title{
The field-induced instability in the planar texture of cholesteric liquid crystals
}

\author{
G. Vertogen \\ Institute for Theoretical Physics, Toernooiveld, Catholic Univeristy of Nijmegen, The Netherlands
}

\section{E. W. C. van Groesen}

Mathematical Institute, Toernooiveld, Catholic University of Nijmegen, The Netherlands (Received 9 September 1981; accepted 16 October 1981)

\begin{abstract}
A more accurate calculation is given of both the striped perturbation pattern and the square grid perturbation pattern that are formed in the planar cholesteric layer as soon as the applied field reaches a threshold value. Notably, it is shown that the distance between the boundaries must exceed a critical value in order to allow such a distortion. The values of the critical field and the wavelength of the distortion are compared with existing calculations.
\end{abstract}

\section{INTRODUCTION}

The planar structure of cholesteric liquid crystals becomes unstable as soon as an applied magnetic field parallel to the helix axis reaches a threshold value. The instability appears in the form of a striped perturbation pattern or a square grid perturbation depending on the number of helical turns between the boundaries of the cholesteric layer. ${ }^{1}$ Approximate expressions for the threshold value of the field and the period of the deformation were obtained by Helfrich ${ }^{2}$ and Hurault. ${ }^{3}$ These expressions were improved by de $\mathrm{Zwart}$ and van Doorn, ${ }^{4}$ who also calculated the influence of pitch contraction and dilation on the static square grid perturbation. The results of de Zwart, and van Doorn, however, hold only for layers with many helical turns and consequently do not apply to the striped perturbation pattern that appear in layers with few turns. This striped perturbation pattern has been dealt with numerically by Chigrinov et al. ${ }^{5}$ The purpose of this paper is to give an analytical treatment of the appearing instability in the planar texture of a cholesteric liquid crystal for all possible helical turns. In order to avoid unnecessarily complicated mathematics we confine ourselves mainly to the one-constant approximation. The general case can be treated analogously. Finally we compare our results with those mentioned above.

\section{THE RELEVANT EQuATIONS}

The starting point is a cholesteric planar layer of thickness $d$ with rigid boundary conditions. The normal to the layer is taken to be parallel to the $z$ axis. At the boundaries both the $y$ and $z$ components of the director $\mathbf{n}$ are zero. Next a magnetic field is applied perpendicular to the layer, i.e., parallel to the $z$ axis. The Frank free energy for a cholesteric in a magnetic field is given by

$$
\begin{aligned}
F= & \frac{1}{2} \int d \mathbf{r}\left[K_{11}(\operatorname{div} \mathbf{n})^{2}+K_{22}\left(n \cdot \operatorname{rot} \mathbf{n}+t_{0}\right)^{2}\right. \\
& \left.+K_{33}(\mathbf{n} \times \operatorname{rot} \mathbf{n})^{2}-\Delta \times(\mathbf{n} \cdot \mathbf{H})^{2}\right],
\end{aligned}
$$

where $K_{11}, K_{22}$, and $K_{33}$ are the Frank elastic constants of splay, twist, and bend, respectively, $\Delta \chi$ is the diamagnetic anisotropy and $\mathrm{H}$ denotes the magnetic field. The natural twist $t_{0}$ is related to the natural pitch $p_{0}$ through $t_{0}=2 \pi / p_{0}$. In the following we consider only the case $t_{0}>0$. Analogous results are obtained if the handedness of the helix is changed. The components of the director $\mathbf{n}$ are given by

$$
\begin{aligned}
& n_{x}=\cos \varphi \cos \psi, \\
& n_{y}=\sin \varphi \cos \psi, \\
& n_{z}=\sin \psi,
\end{aligned}
$$

where $\psi$ is the angle between the director and its projection on the $x y$ plane, which is parallel to the layer, and $\varphi$ is the angle between the projection of $\mathbf{n}$ on the $x y$ plane and the $x$ axis. The boundaries of the layer are defined by the planes $z=0$ and $z=d$. Substitution of the components of $n[E q .(2.2)]$ in the expression for the Frank free energy expresses the distortion in terms of the angles $\varphi$ and $\psi$, that, in the first instance, are functions of the coordinates $x, y$, and $z$. This functional dependence on $x, y$, and $z$ is determined by minimizing the Frank free energy with respect to $\varphi$ and $\psi$. The resulting Euler-Lagrange equations are a system of two coupled nonlinear partial differential equations. In order to determine the critical field it suffices to linearize this coupled system around $\psi=0$ and $\varphi=t z$, which is the solution below threshold. It holds $t=m q$ with $q$ $=\pi / d$, while $m$ is an integer such that $\left(t-t_{0}\right)^{2}$ is minimal. The number $m$ denotes the number of helical turns, where we define a helical turn as a $\pi$ rotation of the director. We remark here that de Zwart and van Doorn define a helical turn as a $2 \pi$ rotation of the director. Putting

$$
\varphi=t z+\xi,
$$

the following system of coupled partial differential equations results

$$
\begin{array}{r}
K_{11} \psi_{z z}+\frac{1}{2}\left(K_{22}+K_{33}\right)\left(\psi_{x x}+\psi_{y y}\right)+\frac{1}{2}\left(K_{33}-K_{22}\right) \cos 2 t z\left(\psi_{x x}-\psi_{y y}\right) \\
+\left(K_{33}-K_{22}\right) \sin 2 t z \psi_{x y}+\left(K_{22}-K_{11}\right)\left(\sin t z \xi_{x z}-\cos t z \xi_{y z}\right) \\
-\left[\left(K_{33}+K_{11}-2 K_{22}\right) t+2 K_{22} t_{0}\right]\left(\cos t z \xi_{x}+\sin t z \xi_{y}\right)+\Lambda \psi=0 \\
(2.4 \mathrm{a}) \\
K_{22} \xi_{z z}+\frac{1}{2}\left(K_{11}+K_{33}\right)\left(\xi_{x x}+\xi_{y y}\right)+\frac{1}{2}\left(K_{33}-K_{11}\right) \cos 2 t z\left(\xi_{x x}-\xi_{y y}\right) \\
+\left(K_{33}-K_{11}\right) \sin 2 t z \xi_{x y}+\left(K_{22}-K_{11}\right)\left(\sin t z \psi_{x z}-\cos t z \psi_{y z}\right) \\
+\left[\left(K_{33}-K_{22}\right) t+2 K_{22} t_{0}\right]\left(\cos t z \psi_{x}+\sin t z \psi_{y}\right)=0, \quad(2.4 \mathrm{~b})
\end{array}
$$


where

$$
\Lambda=\Delta \chi H^{2}+2 K_{22} t\left(t-t_{0}\right)-K_{33} t^{2}
$$

and

$$
\alpha_{k}=\frac{\partial \alpha}{\partial k} ; \quad \alpha_{k l}=\frac{\partial^{2} \alpha}{\partial k \partial l} ; \alpha=\psi, \xi, \quad k, l=x, y, z .
$$

The solution of this system has to satisfy the boundary conditions

$$
\psi(z=0)=\psi(z=d)=\xi(z=0)=\xi(z=d)=0 .
$$

The critical field $H_{c}$ corresponding with the onset of the deformation is obtained by determining the lowest eigenvalue $\Lambda$.

\section{THE DETERMINATION OF THE LOWEST EIGENVALUE}

The system of coupled partial differential equations (2.4) allows for three different types of solutions, viz. (a) the Leslie solution, ${ }^{6}$ (b) a helixlike solution with the helix axis in the $x$ direction, and (c) a helixlike solution with its helix axis in the $y$ direction. (A) First we consider the Leslie solution in which the distortion depends only on $z$, i.e., $\psi(x, y, z)=f(z)$ and $\xi(x, y, z)=g(z)$. The functions $f(z)$ and $g(z)$ satisfy the following system of differential equations

$$
\begin{aligned}
& K_{11} f_{z z}+\Lambda f=0, \\
& K_{22} g_{z z}=0,
\end{aligned}
$$

and the boundary conditions $f(o)=f(d)=g(o)=g(d)=0$. Clearly the solution is given by $g(z) \equiv 0$ and $f(z)=$ $\sin (n q z), n=0,1,2, \ldots$. Thus the critical field of the Leslie distortion follows from $\Lambda=K_{11} q^{2}$ or

$$
\Delta \chi H_{c}^{2}=K_{33} t^{2}+K_{11} q^{2}+2 K_{22} t\left(t_{0}-t\right) .
$$

The corresponding critical field for a nematic is given by $\Delta \chi H_{c}^{2}=K_{11} q^{2}$. Therefore changing the nematic into a cholesteric with pitch $p_{0}$ will raise the original critical field by a factor

$$
1+4 \frac{K_{33}}{K_{11}}\left(\frac{d}{p}\right)^{2}+8 \frac{K_{22}}{K_{11}}\left(\frac{d}{p}\right)\left[\left(\frac{d}{p}\right)-\left(\frac{d}{p_{0}}\right)\right],
$$

where $p$ denotes the actual pitch of the cholesteric, i.e., the pitch imposed by the boundary conditions. (B) Next we consider the helixlike solution with its helix axis in the $x$ direction. This Helfrich-Hurault type of solution can be written as

$$
\begin{aligned}
& \psi(x, y, z)=f(z) \cos k x, \\
& \xi(x, y, z)=g(z) \sin k x,
\end{aligned}
$$

with $f(o)=f(d)=g(o)=g(d)=0$. In the one-constant approximation, i.e., $K_{11}=K_{22}=K_{33}=K$, the functions $f(z)$ and $g(z)$ fulfill the following system of coupled differential equations

$$
\begin{aligned}
& f_{z z}-k^{2} f+\lambda f-2 k t_{0} \cos t z \cdot g=0, \\
& g_{z z}-k^{2} g-2 k t_{0} \cos t z \cdot f=0,
\end{aligned}
$$

with $K \lambda=\Lambda$. The natural way to take the boundary conditions into account is by representing $f(z)$ and $g(z)$ as a Fourier series. Then we are faced with the problem of solving an infinite system of coupled linear equations in the Fourier coefficients. Our interest, however, does not concern the general solution of such a system but only the lowest eigenvalue. By expressing the eigenvalue $\lambda$ in terms of the Fourier coefficients of the functions $f(z)$ and $g(z)$ we can determine an upper bound for the lowest eigenvalue. A reasonable approximation of this lowest eigenvalue can be obtained by approximating $g(z)$ by its first Fourier component that satisfies the boundary condition, i.e., $g(z)=\sin q z$. For it should be expected that the helix itself should be only slightly distorted just above threshold. Then it follows from Eqs. (3.5) that an approximate expression for the lowest eigenvalue can be obtained by solving

$$
\begin{aligned}
& -\left\langle f_{z}, f_{z}\right\rangle-k^{2}\langle f, f\rangle+\lambda\langle f, f\rangle \\
& \quad-2 k t_{0}\langle f, \cos t z \cdot \sin q z\rangle=0, \\
& -\frac{1}{2}\left(q^{2}+k^{2}\right)-2 k t_{0}\langle\sin q z, \cos t z \cdot f\rangle=0,
\end{aligned}
$$

where we used the notation

$$
\left\langle h_{1}, h_{2}\right\rangle=\frac{1}{d} \int_{0}^{d} h_{1}(z) h_{2}(z) d z,
$$

and $\left\langle f, f_{z z}\right\rangle=-\left\langle f_{z}, f_{z}\right\rangle$. Because the case $m=1$ differs slightly from the general case we treat this case separately.

The case $m=1$. Put $t=q$ and $f(z)=a \cos q z \cdot \sin q z$. Then we find

$$
\lambda=4 q^{2}+k^{2}-\frac{k^{2} t_{0}^{2}}{q^{2}+k^{2}} .
$$

The wave number $k$ is determined by the requirement that $\lambda$ should be minimal, i.e., $\partial \lambda / \partial k^{2}=0$. Then it follows that

$$
\begin{aligned}
k^{2} & =q\left(t_{0}-q\right), & & t_{0}>q, \\
& =0, & & t_{0}<q .
\end{aligned}
$$

It is easily verified that the resulting threshold exceeds the Leslie threshold.

The general case. Put $t=m q$ and $f(z)=a(\cos m q z$

- $\left.\sin q z-\sigma \sin m q z^{\cdot} \cos q z\right)$. Then we find

$$
\begin{aligned}
\lambda= & m^{2} q^{2}+q^{2}+k^{2} \\
& -4 m q^{2} \frac{\sigma}{1+\sigma^{2}}-2 \frac{k^{2} t_{0}^{2}}{\left(q^{2}+k^{2}\right)\left(1+\sigma^{2}\right)} .
\end{aligned}
$$

The parameter $\sigma$ is determined by the requirement that it should minimize $\lambda$. It follows that:

$$
\sigma=\frac{1}{2 m q^{2}}\left\{\left[4 m^{2} q^{4}+\frac{k^{4} t_{0}^{4}}{\left(q^{2}+k^{2}\right)^{2}}\right]^{1 / 2}-\frac{k^{2} t_{0}^{2}}{q^{2}+k^{2}}\right\} .
$$

Substitution of Eq. (3.11) into Eq. (3.10) gives

$$
\begin{aligned}
\lambda= & m^{2} q^{2}+q^{2}+k^{2} \\
& -\frac{k^{2} t_{0}^{2}}{q^{2}+k^{2}}-\left[4 m^{2} q^{4}+\frac{k^{4} t_{0}^{4}}{\left(q^{2}+k^{2}\right)^{2}}\right]^{1 / 2} .
\end{aligned}
$$

The wave number $k$ is determined by minimizing $\lambda$ with respect to it.

If $m$ is large the Eq. (3.12) can be approximated by

$$
\lambda=m^{2} q^{2}+q^{2}+k^{2}-\frac{2 k^{2} t_{0}^{2}}{q^{2}+k^{2}} .
$$


In this case we may put $\sigma=0$. The expression for the wave number $k$ is then given by

$$
k^{2}=q\left(\sqrt{2} t_{0}-q\right) \text {. }
$$

This means that the threshold value of the field follows from

$$
\lambda=m^{2} q^{2}+2 \sqrt{2} q t_{0}-2 t_{0}^{2} .
$$

Equation (3.12) gives always rise to a threshold value which is lower than the Leslie threshold. On the other hand Eq. (3.15) gives rise to a threshold lower than the Leslie threshold provided that $t_{0}>\frac{1}{2} \sqrt{2}(m+1) q$. We require that the approximation holds for the total region $\left(m-\frac{1}{2}\right) q<t_{0}<\left(m+\frac{1}{2}\right) q$. This means that the approximation can be used provided that $m \geqslant 5$. (C) Finally we consider the helixlike solution with its helix axis in the $y$ direction. This type of solution can be written like

$$
\begin{aligned}
& \psi(x, y, z)=r(z) \cos (l y), \\
& \xi(x, y, z)=s(z) \sin (l y),
\end{aligned}
$$

with $r(o)=r(d)=s(o)=s(d)=0$. In the one-constant approximation the functions $r(z)$ and $s(z)$ fulfill the following system of coupled differential equations

$$
\begin{aligned}
& r_{z z}-l^{2} r+\lambda r-2 L t_{0} \sin t z \cdot s=0, \\
& s_{z z}-l^{2} s-2 l t_{0} \sin t z \cdot r=0 .
\end{aligned}
$$

Analogous to the previous case an approximate expression for the lowest eigenvalue can be obtained by solving $-\left\langle r_{z}, r_{z}\right\rangle-l^{2}\langle r, r\rangle+\lambda\langle r, r\rangle-2 l t_{0}\langle r, \sin t z \cdot \sin q z\rangle=0$,

$$
-\frac{1}{2}\left(q^{2}+l^{2}\right)-2 l t_{0}\langle\sin q z, \sin t z \cdot r\rangle=0,
$$

where $s(z)$ has been approximated by $\sin q z$. Because here also the case $m=1$ differs from the general case we deal with that case separately.

The case $m=1$. Put $t=q$ and $r(z)=b \sin q z$. Then we find

$$
\lambda=q^{2}+l^{2}-\left(\frac{16}{3 \pi}\right)^{2} \frac{l^{2} t_{0}^{2}}{q^{2}+l^{2}},
$$

where the wave number $l$ is given by

$$
l^{2}=q\left(\frac{16}{3 \pi} t_{0}-q\right) \text {. }
$$

This means that a helixlike solution is only possible provided that the distance $d$ between the boundaries exceeds a critical distance $d_{c}$ given by

$$
d_{c}=\frac{3 \pi}{32} p_{0} \simeq 0.29 p_{0} .
$$

The threshold value of the field follows from

$$
\lambda=\frac{32}{3 \pi} q t_{0}-\left(\frac{16}{3 \pi}\right)^{2} i_{0}^{2} .
$$

Obviously the threshold for this type of distortion is lower than the Leslie threshold.

The general case. Put $t=m q$ and compare the results of the trial functions (a) $r(z)=a \sin m q z$; (b) $r(z)$

$=a \sin m q z \cdot \sin q z$. Substitution of the trial function $r(z)$ $=a \sin m g z$ in $\mathrm{Eq}$. $(3.18)$ gives rise to

$$
\lambda=m^{2} q^{2}+l^{2}-\left[\frac{16 m^{2}}{\pi\left(4 m^{2}-1\right)}\right]^{2} \frac{l^{2} t_{0}^{2}}{q^{2}+l^{2}},
$$

where the wave number $l$ is given by

$$
l^{2}=q\left[\frac{16 m^{2}}{\pi\left(4 m^{2}-1\right)} t_{0}-q\right] \text {. }
$$

The threshold belonging to this distortion is given by

$\lambda=m^{2} q^{2}-q^{2}+\frac{32 m^{2}}{\pi\left(4 m^{2}-1\right)} q t_{0}-\left(\frac{16 m^{2}}{\pi\left(4 m^{2}-1\right)}\right)^{2} t_{0}^{2}$,

and is lower than the Leslie threshold provided that

$$
t_{0}>\frac{\pi\left(4 m^{2}-1\right)}{16 m^{2}}\left[1+\left(m^{2}-1\right)^{1 / 2}\right] q \text {. }
$$

(b) Substitution of trial function $\gamma(z)=a \sin m q z \cdot \sin q z$ results in

$$
\lambda=\left(m^{2}+1\right) q^{2}+l^{2}-\frac{2 l^{2} t_{0}^{2}}{q^{2}+l^{2}},
$$

with

$$
l^{2}=q\left(\sqrt{2} t_{0}-q\right) .
$$

This means that the threshold value of the field follows from

$$
\lambda=m^{2} q^{2}+2 \sqrt{2} t_{0} q-2 t_{0}^{2} .
$$

Both trial functions give rise to a higher threshold than the corresponding helixlike distortion in the $x$ direction. For large $m$ the threshold value corresponding with the trial function (b) is lower than the threshold value corresponding with the trial function (a) and approaches the threshold value of the corresponding helixlike solution with its helix axis in the $x$ direction.

\section{CONCLUSION}

Our results clearly indicate that in the case of one helical turn, i.e., $m=1$, a striped perturbation pattern is obtained as soon as the distance between the boundaries exceeds the critical distance $d_{c}=(3 \pi / 32) p_{D}$. If the distance is smaller than the critical distance the instability is the Leslie distortion. The striped $m=1$ perturbation pattern is due to a helixlike distortion with the helix axis in the $y$ direction. In the case of more helical turns, $m>1$, a striped perturbation pattern is obtained being perpendicular to the $m=1$ pattern and due to a helixlike distortion with its helix axis in the $x$ direction. The threshold value of the critical field for a helixlike distortion with its helix axis in the $y$ direction appears to be slightly higher. With increasing $m$, however, the threshold values for both types of distortion converge strongly to each other. This is the reason why a square grid perturbation is abserved.

In order to compare our results with the calculations of de $Z$ wart and van Doorn $n^{4}$ we give here the expression for the critical magnetic field $H_{c}$ using Eqs. (3.2) and (3.15). We have

$$
\left(\frac{H_{\varepsilon}}{H_{0}}\right)^{2}=4 \sqrt{2} \frac{d}{p_{0}}+8\left(\frac{d}{p}-\frac{d}{p_{0}}\right) \frac{d}{p_{0}},
$$

with

$$
H_{0}^{2}=\left(\frac{\pi}{d}\right)^{2} \frac{K}{\Delta \chi} .
$$


The wavelength $\lambda$ of the distortion is given by

$$
\lambda=\left[\frac{4 \sqrt{2} p_{0} d}{4 d-\sqrt{2} p_{0}}\right]^{1 / 2} \text {. }
$$

The corresponding expressions in the slightly improved de Zwart and van Doorn calculations ${ }^{1}$ read in the oneconstant approximation

$$
\left(\frac{H_{c}}{H_{0}}\right)^{2}=4 \sqrt{2} \frac{d}{p}+8\left(\frac{d}{p}-\frac{d}{p_{0}}\right) \frac{d}{p}
$$

and

$$
\lambda=[\sqrt{2} p d]^{1 / 2} .
$$

Clearly the results of de $\mathrm{Z}$ wart and van Doorn are quite reliable in the case of many helical turns, i.e., $d / p$ is large.

Finally we give the value of the critical field in the general case for large values of $m$, i.e., many helical turns. Using the relevant trial functions, i.e., $f(z)$ $=a \cos m q z \cdot \sin q z$ for a helixlike distortion with its helix axis in the $x$ direction and $r(z)=a \sin m q z \cdot \sin q z$ for a helixlike distortion with its helix axis in the $y$ direction, we obtain the following value for the critical field $H_{c}$ :

$$
\begin{aligned}
\Delta \times H_{c}^{2}= & K_{11} q^{2}+\frac{1}{4}\left(3 K_{33}+K_{22}\right) k^{2} \\
& +K_{22} \frac{\left[\left(K_{33}+K_{11}-2 K_{22}\right) t+2 K_{22} l_{0}\right]\left[q^{2} t+\left(\ell-\ell_{0}\right) k^{2}\right]}{K_{22} q^{2}+\frac{1}{2}\left(K_{11}+K_{33}\right) k^{2}} .
\end{aligned}
$$

The wavelength of the distortion is obtained by minimizing the value of the critical field $H_{c}$ with respect to $k$. After some algebra the following expression results:

$$
\begin{aligned}
k^{2}= & \frac{2\left[\left(K_{11}+K_{33}-2 K_{22}\right) t+2 K_{22} t_{0}\right]}{K_{11}+K_{33}} q \\
& \times\left[\frac{2 K_{22}}{K_{22}+3 K_{33}}\right]^{1 / 2}-\frac{2 K_{22}}{K_{11}+K_{33}} q^{2} .
\end{aligned}
$$

When Eq. (4.7) is substituted in Eq. (4.6) and $q, t$, and $t_{0}$ are expressed in $d, p$, and $p_{0}$, respectively, the following equation is obtained:

$$
\begin{aligned}
\left(\frac{H_{c}}{H_{0}}\right)^{2}= & \frac{K_{11}}{K_{22}}-\frac{3 K_{33}+K_{22}}{2\left(K_{11}+K_{33}\right)}+2\left[\frac{3 K_{33}+K_{22}}{K_{22}}\right]^{1 / 2} \frac{d}{p}+2\left[\frac{3 K_{33}+K_{22}}{K_{22}}\right]^{1 / 2} \frac{\left[\left(K_{11}+K_{33}-2 K_{22}\right)+2 K_{23}(p / p 0)\right]}{\left(K_{11}+K_{33}\right)} \frac{d}{p} \\
& +8 \frac{\left[\left(K_{11}+K_{33}-2 K_{22}\right)+2 K_{22}\left(p / p_{0}\right)\right]\left[1-\left(p / p_{0}\right)\right]}{\left(K_{11}+K_{33}\right)}\left(\frac{d}{p}\right)^{2}-\frac{\left[2 K_{22}\left(3 K_{33}+K_{22}\right)\right]^{1 / 2}}{K_{11}+K_{33}}\left(1-\frac{p}{p_{0}}\right) \frac{d}{p}
\end{aligned}
$$

with

$$
H_{0}^{2}=\left(\frac{\pi}{d}\right)^{2}\left(\frac{K_{22}}{\Delta \chi}\right) .
$$

The wavelength of the perturbation is given by

$$
\lambda^{2}=\left(\frac{3 K_{33}+K_{22}}{2 K_{22}}\right)^{1 / 2} p d\left[\frac{\left(K_{11}+K_{33}-2 K_{22}\right)+2 K_{22}\left(p / p_{0}\right)}{K_{11}+K_{33}}-\frac{K_{22}}{2\left(K_{11}+K_{33}\right)} \frac{p}{d}\left(\frac{3 K_{33}+K_{22}}{2 K_{22}}\right)^{1 / 2}\right]^{-1 / 2} .
$$

We remark here that Eqs. (4.8) and (4.9) do not agree with the equations derived by Hurault ${ }^{3}$ by putting $p=p_{0}$.

\section{ACKNOWLEDGMENTS}

We would like to thank Dr. M. de Zwart of Videlec, Dr. F. Leenhouts of Siemens A. G., and Dr. C. Z. van Doorn and Dr. W. J. A. Goossens of the Philips Research Laboratories for stimulating discussions.

${ }^{1}$ M. de Zwart, Thesis, Nijmegen, 1981.
${ }^{2}$ W. Helfrich, Appl. Phys. Lett. 17, 531 (1970); W. Helfrich, J. Chem. Phys. 55, 839 (1971).

${ }^{3}$ J. P. Hurault, J. Chem. Phys. 59, 2068 (1973).

${ }^{4}$ M. de Zwart and C. Z. van Doorn, J. Phys. C 40,3278 (1979).

${ }^{5}$ V. G. Chigrinov, V. V. Belyaev, S. V. Belyaev, and M. F. Grebenkin, Sov. Phys. JETP 50, 994 (1979).

${ }^{6}$ F. M. Leslie, Mol. Cryst. Liq. Cryst. 12, 57 (1970). 